\title{
Translational Medicine: Challenges and new orthopaedic vision (Mediouni-Model)
}

\author{
Mohamed Mediouni (1*), Riadh Madiouni (2), Michael Gardner (3), Neil Vaughan (4) \\ (1) Independent Researcher, Sherbrooke, Québec, Canada. \\ (2) The Images, Signals and Intelligent Systems Laboratory, LiSSi-EA-3956, University of Paris-Est Créteil, 122 rue \\ Paul Armangot, 94400 Vitry sur Seine, France \\ (3) Department of Orthopaedic Surgery, Stanford University, USA \\ (4) University of Chester, Chester, UK \\ (*) Correspondence author: Mohamed Mediouni, Independent Researcher, Sherbrooke, 1420 rue Laterierre, \\ Sherbrooke, Quebec, J1K 2R2 Canada Tel: +1 8738887263; E-mail: Mohamed.Sherbrooke@gmail.com
}

\begin{abstract}
Background: In North America and three European countries Translational Medicine (TM) funding has taken center stage as the National Institutes of Health (NIH), for example, has come to recognize that delays are common place in completing clinical trials based upon benchside advancements. Recently, there are several illustrative examples whereby the translation of research had untoward outcomes requiring immediate action.
\end{abstract}

Methods: Focus more on three-dimensional (3D) simulation, biomarkers, and Artificial Intelligence may allow orthopaedic surgeons to predict the ideal practices before orthopaedic surgery. Using the best medical imaging techniques may improve the accuracy and precision of tumor resections.

Results: This article is directed at the young surgeon scientist and in particular orthopaedic residents and all other junior physicians in training to help them better understand TM and position themselves in career paths and hospital systems that strive for optimal TM. It serves to hasten the movement of knowledge garnered from the benchside and move it quickly to the bedside.

Conclusions: Communication is ongoing in a bidirectional format. It is anticipated that more and more medical Centers and institutions will adopt TM models of healthcare delivery.

Keywords: Translational Medicine; 3D simulation; Tumor bone, biomarkers, Artificial Intelligence 


\section{Introduction}

Translational Medicine (TM) has received significant attention in the past decade and it is still a buzzword in the medical field today. Given the growing impact of scientific discoveries, translational medicine was initially described as the 'marriage' between basic science and clinical science which represents a tremendous challenge $[\mathbf{1 , 2 , 3 , 4 ]}$. What defines TM? According to the European Society for Translational Medicine (EUSTM) TM consists of 3 pillars: the scientist at his/her benchside; the clinician at the bedside; and the community at large, which includes the drivers of medical advancements such as non-profit foundations, government funded universities and corporations providing medical services devices or medications within house research and development $(\mathrm{RD})$ divisions. The interaction between the 3 pillars is bidirectional ("bench to bedside" and "bedside to bench to bedside"). The main objective of translational research from "bench to bedside" is to test in human novel discoveries. Based on basic science such as mathematics, physics, biology and chemistry, we can produce new drugs, devices and treatments for patients. Sometimes, our understanding of many human diseases, such as hypertension is very limited. For that purpose, there is a critical need for "bedside to bench to bedside". Ernesto et al. [5] give an excellent example of bedside to bench to bedside. The authors explain the role of renin-angiotensin-aldosterone system in remodeling of resistance arteries in hypertension. To conclude, the global objective of TM is to advance local or regional med-surg care swiftly successfully, and repeatedly and then apply these methods on a broader scale to improve sovereign, national and

eventually global healthcare. All of this is in direct contrast to traditional academic centers with laboratories and professors working independently within their own discipline with little to no communication with other scientists. The best possible description of this separatism arrangement is the proverbial silo effect or isolation.

\section{Obstacles to Translational Medicine}

Many challenges face scientists to apply translational research, including lack of sufficient funding, high cost and slow results, and lack of congressional and public support $[6,7,8,9,10]$. In the case of the bench-to-bedside direction, the complexity of translational research in human subjects is overwhelming: production and validation of products of consistent safety, potency and quality, the ancillary needs associated with the care of patients with severe conditions and the cost of validating translatable biomarkers renders this discipline uniquely expensive. How can small or medium biotechnology firms justify these costs? Similarly, how can academic institutions seeking to translate ideas of their research faculty take the economical risk of supporting a promising clinical trial? Most translational research is supported by grants; however, few provide funding for regulatory staff or consultation. Academic institutions do not provide support to non hypothesis driven research such as product development. Translational researchers need special training in the complexity of new technologies. Dual-trained physician-scientists are too few. For that purpose we need to focus on the development of scientific training programs for clinicians $[\mathbf{1 1}, \mathbf{4 1}]$. Academic physicians in training should spend time in the laboratory and consider graduate courses or seminars that update and deepen their understanding of current research 
[46]. The purpose of these initiatives is not to increase the number of individuals who are fully trained in biology, math, medicine, statistics, etc [12].

\section{Mediouni-Model (T-model): What does it involve?}

In literature, many models have been used to improve the practice of surgeons in the operating room, such as Gawande [13], Howell [14], Miller [15] and Sadideen [16]. In 2019, Mediouni proposed a T-model that aims to bridges the gap between basic science and clinical sciences [17] (figure 1). The success of this model is based on multidisciplinary teams, which are involved in translational orthopaedics. Collaboration among disciplines through multidisciplinary teams facilitates the emergence of novel concepts. For example, the surgeon who works on bone tissue regeneration will participate in the activities of a clinical orthopedic surgery program and will discuss techniques of molecular biology or genetics. Based on those activities, surgeons will learn thinking skills, which help her/him to criticize new practices.

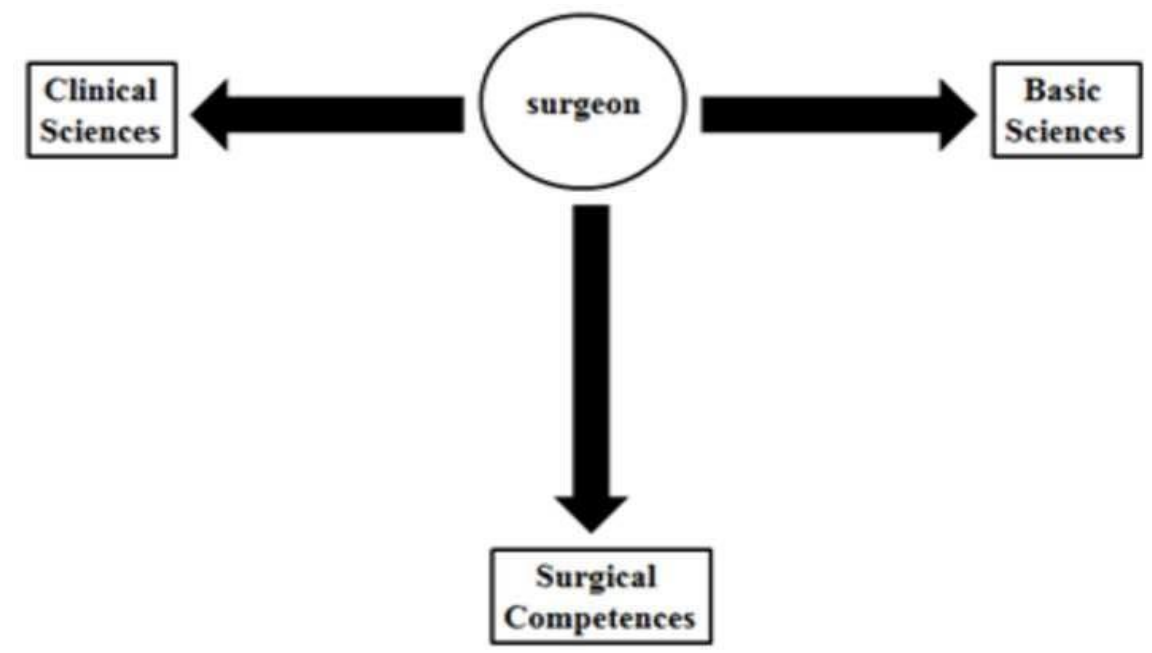

Figure 1. Mediouni Model or (T-model).

A deeper understanding of the cellular and molecular mechanisms that mediates bone formation can accelerate the treatment of complicated operation. For example, skeleton is one of the most structure in our body. Understanding more the microarchitecture can improve the treatment of osteoporosis which represents a global health problem.

This new strategy can accelerate the development of artificial joints and limbs to relieve pain and improve quality of life, It can help the treatment of injuries arising from accidents or sporting activities.

Translational orthopaedic is suitable for projects that build on orthopaedic science to develop therapies that may reach the clinical stage. These projects are intended to create the value of the associated intellectual property through activities such as prototype development and testing for proof of concept. 


\section{The Role of Three-Dimensional (3D) Simulation in Orthopaedic practice}

New approaches simulation are emerging that bring computer science, physics, chemistry and engineering. To radically improve our understanding of medical devices, simulation offers many benefits for designing and understanding mechanical behaviour of devices. There are several key design and simulation approaches available to researchers, engineers, and analysts, including Computer-Aided Design (CAD). Finite Element Analysis (FEA) $[18,19]$ is a numerical method which can calculates stresses and forces during the contact between structures. Using FEA, to avoid the problems of osteonecrosis, we can measure the temperature by estimating the parameters during drilling such as: speed of drill bit, feed rates, forces applied by the users (see figure 2) [20].
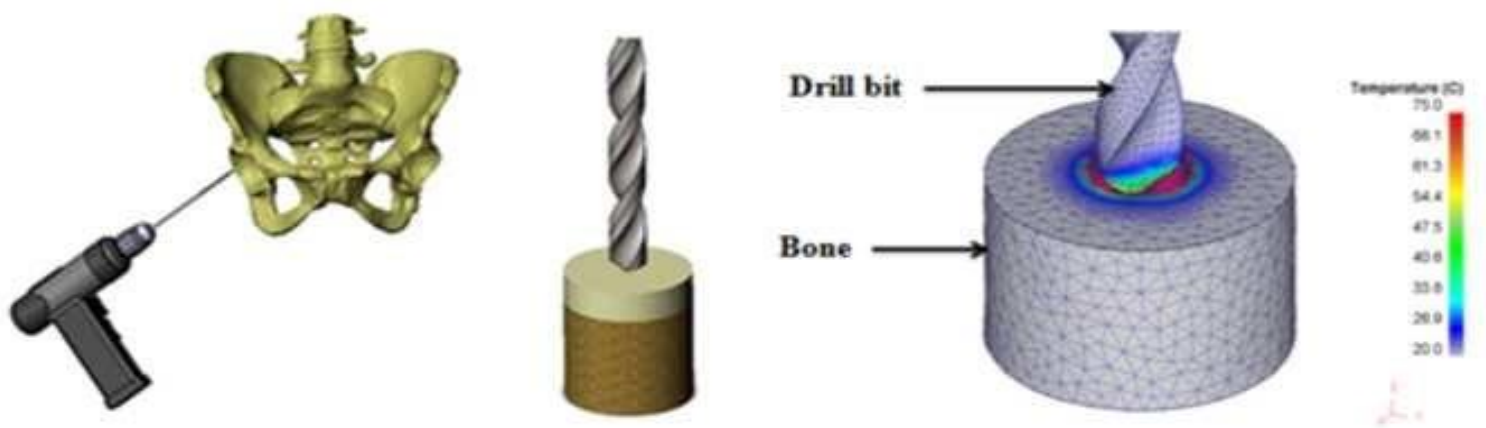

Figure 2: (a) 3D simulation of drilling of acetabular bone, (b) A pelvic model and FEA defined variables to reduce heat generation and its consequence (threshold is $50^{\circ} \mathrm{C}$ ), bone necrosis. The FEA data has two never before reported findings. (1) When drilling $3 \mathrm{~mm}$ of cortical bone at a constant rotation of $600 \mathrm{rev} / \mathrm{min}$. feeding $1 \mathrm{~mm} / \mathrm{sec}$ produces a force of 50-80 Newton (N) and $75^{\circ} \mathrm{C}$ temperature, and (2) Reducing the feeding speed to $0.1 \mathrm{~mm} / \mathrm{sec}$, the temperature is reduced to $39^{\circ} \mathrm{C}$, with constant axial force and rotation of $600 \mathrm{rev} / \mathrm{min}$. Extracted from [20].

Computational Fluid Dynamics (CFD) [21] is another branch that allows one to simulate fluid motion using numerical approaches. CFD may address many challenges and answers for example the question: What is the best volume of water at room temperature to minimize bone cell death by keeping the metal drill bit temperature low? In the literature, there is no application in orthopaedics which combine CFD and FEA. Acquiring three-dimensional (3D) imaging data from medical images magnetic resonance imaging (MRI) and Computed Tomography (CT) provides a basis for creating anatomical models of human anatomy. The 3D model image data are used in many applications, including analyzing implant stability and tissue stress. Many challenges faced orthopaedic simulation in order to reproduce the complex structures include technical competences, consistency between results, and validation. Being able use the simulator for training can help surgeons to improve their practice. Frankly, simulation does not replace experimental testing, but provides a fast-evolving option for validation and evaluation. 


\section{The Role of Imaging for TM in Bone Tumors}

Cancer is responsible for the death of many people around the world [44]. A large percentage of patients die after developing cancer despite aggressive treatment. There is a great need for new approaches to cancer therapy. Genomic and protein technology have generated a huge amount of essential information to broaden our understanding of cancer biology. New research has paved the way for drug development targeting biological and specific models. Introduction of imatinib into the clinic took forty years beyond the discovery of the Philadelphia chromosome [22]. Trastuzumab was not clinically available for another twenty years and faced considerable difficulties throughout its development and testing process. A possible explanation of the gap between clinical and preclinical results is the interaction between targeted molecular therapy and cytotoxic targeting the same tumor cell. The main goal of translational research is to design the best methods and strategies that allow for high efficiency. The development of new images techniques can have a major impact on the practice of oncology. Quantitative dynamic-contrast magnetic resonance (MR) imaging is a non-invasive technique that estimates the percentage of necrosis in bone tumors and post-treatment modification [23]. It incorporates two approaches for the acquisition of dynamic MR images such as DCE-MR imaging use T1-weighted acquisitions, while susceptibility based techniques such as dynamic susceptibility contrast-enhanced MR imaging (DSC-MR imaging) use T2-based sequences [24].

Diffusion Weighted Imaging (DWI) [25] is generally used to visualize areas of tissue in which a pathological process has altered the movement of fluid that is outside the vessels. Water diffusion is more limited in the tumor region than in normal tissues. This information can represent a high signal intensity. Today, the progression of molecular imaging allows clinicians to understand tumor biology better. For

example, optical imaging [26] is based on light emission following enzymatic reaction of luciferase which is used to see the tumor's progression (see figure 3). Magnetic resonance spectroscopy (MRS) [27] is another modality which has advantages compared to other modalities, including the improvement of signal/noise ratio, spatial resolution and the possibility to obtain anatomical and physiological information. It allows measurement of the quantity of metabolites which can reflect molecular composition of tumor.

Today, several combination strategies are used in translational studies. Combinations include: MRI/optical imaging, nuclear imaging / optical imaging, and Nuclear imaging/ MRI. By combining different modalities, additive information can be used to evaluate the progression of the tumors compared to using a single imaging modality alone [28]. However, the clinical translation of these combinations system could be difficult. The integrated system design for preclinical and clinical scanners should be significant. A better understanding of cancer gene as well as the growing superiority of imaging technologies helps crucial researchers and clinicians in translating the disease. 


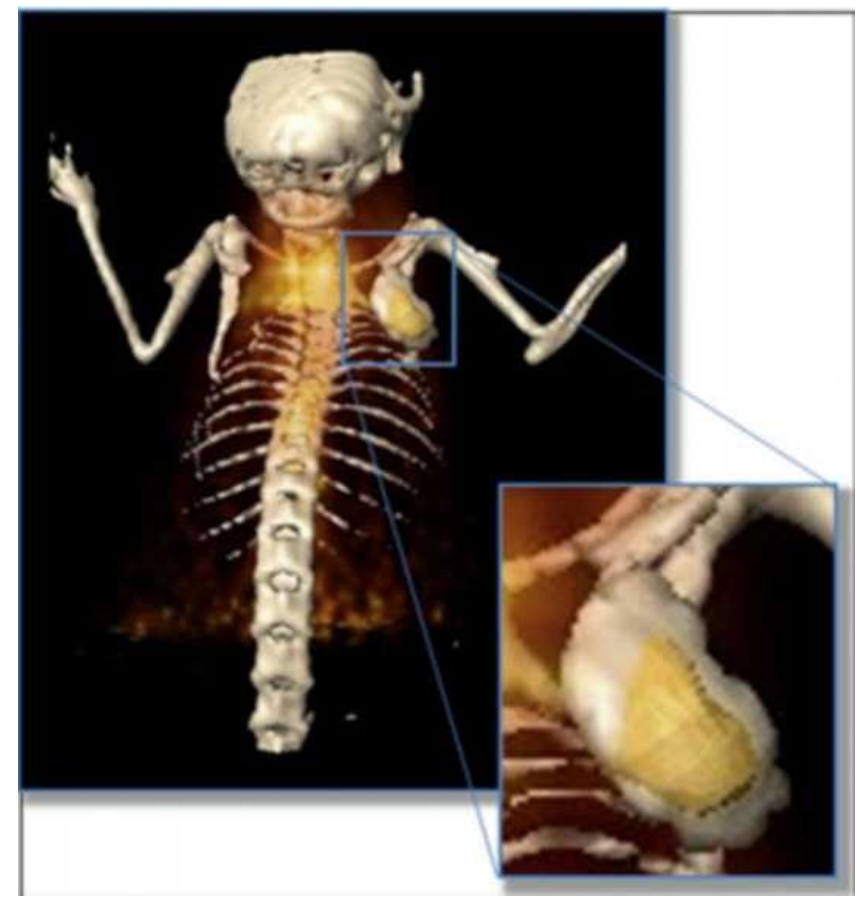

Figure 3. Multimodality imaging of Balb/C athymic nude mouse bearing human MCF7 tumor xenograft [26].

\section{Biomarkers to improve orthopaedic results}

Biomarkers open new perspectives for diagnosis. That they are genetic, physiological and detectable in the blood, biopsy, or imaging, measurable biological features, called biomarkers, act as indicators of the onset of disease or susceptibility to certain conditions. Advances in genetic, protein and cellular analysis techniques, especially since the sequencing of the human genome in 2003, encourage researchers to analyze the mechanisms underlying the development of a disease. It is then necessary to identify the variations that will make it possible to distinguish the healthy individual from the patient. In other words, we are looking for potential biomarkers, heralding an orthopaedic problem. We can cite the role of biomarkers in the diagnostic of periprosthetic joint infection (PJI). The diagnostic of PJI is very difficult and the clinicians have to use a combination of tests that are not accurate [29]. In literature, several molecular biomarkers aimed to analyze the current diagnostic measures for PJI such as serum biomarkers [30] and Synovial fluid biomarkers [31]. The study of [32] shown that serum markers namely CRP and ESR, are elevated with any type of inflammation and infection, compromising their specificity for the diagnosis of PJI. Synovial fluid biomarkers play a very important role in the diagnosis of PJI. When infection occurs in a joint, cytokines such as IL-1b, IL-6, IL-8, IL-17, and TNF- $\alpha$ are released from macrophages. The biomarkers can identify Prei-Implant Osteolysis before radiographic diagnosis [33]. It can differentiate patients who developed osteolysis from those who did not develop osteolysis before radiographic diagnosis. Today, Osteoarthritis (OA) is the most common disease affecting the joints in humans and is an important cause of pain, disability [34, 35, 36]. New information and studies in equine musculoskeletal biomarkers have potential translational value 
for humans and vice versa. Mcllwraith et al. [37] explain the biomarkers for equine joint injury and osteoarthritis. In osteosarcoma, the biology of malignant progression is still unknown. Therefore, protein and gene expression studies have been used to provide to obtain clues about the causes of osteosarcomas. The identification of tumor-specific biomarkers and therapeutic targets is the most important challenge to understand and to predict osteosarcoma $[\mathbf{3 8}, \mathbf{3 9}, \mathbf{4 0}]$.

\section{Application of Artificial Intelligence in orthopaedics}

Recently, artificial intelligence (Al) has become more widespread and it's use in medical and orthopaedic applications has been increasing. A recent review of Al in orthopaedics (Panchmatia et al., 2018) [41] summarised that Al in orthopaedics can improve patient care in four key areas: (i) diagnosis, (ii) management, (iii) research and (iv) systems analysis. Specific examples of Al in orthopaedics include personalizing patient care and also enabling high-quality orthopaedic care even within lower resource settings, for example by increasing research capacity within institutions.

Developing efficient and effective Al solutions to apply within orthopaedic disciplines requires multidisciplinary inputs. The Al cannot be developed by computer scientists in isolation but requires ongoing and active input from clinicians. Involving orthopaedic consultants in development of Al ensures achieving maximum benefit for patients [42] and will take into account all necessary health and safety, ethical, governance, legal regulation, and health service management considerations

One area in which Al is impacting to benefit orthopaedic practice is for fracture detection, which incorporates Al algorithms into the medical image analysis (Langerhuizen et al., 2019) [43]. Various clinical studies have investigated this emerging area of orthopaedic Al. Fracture detection and fracture classification has been applied to many bones including spine, wrist, ankle, hand, hip, ulna, diaphyseal femur, and proximal humerus [43] producing near perfect prediction (range, $0.95-$

1.0) which could potentially outperform human experts.

Currently, Al has shown benefit in challenging diagnostic and therapeutic orthopaedic scenarios. Moving forward, standardized methods to train and test Al are required to enable further integration within clinical workflows. The decision making capabilities of Al has been fuelled by growing availability of large datasets, innovative algorithms, and increased storage capacity and lower costs of high computational power. In orthopaedics, Al provides value for health services and relieve time pressures, by lowering reliance on expert consultants, by enabling (i) advanced data discovery extraction (ii) improved predictive and diagnostic capabilities, and (iii) Al driven orthopaedic decision support systems.

\section{Discussion and conclusions}

Working fluently, clinical results could be improved with more awareness concerning the applied and translational characteristics of basic research. Today, we need a new generation of researchers: "translational scientists" who can play a necessary part in coordination the different 
field of research. Elias Zerhouni, the director of the NIH (2002-2008) is an example of the type scientist that we need, he convened a series of whirlwind meetings with top clinicians and scientists. He has a unique perspective on the complex process of bringing drugs to clinical applications (see figure 4). The Senior author MM propose Mediouni model or (T-model) in which the communication between surgeons with other scientists in many fields has many benefits in terms of understanding a solutions for complex clinical problems.

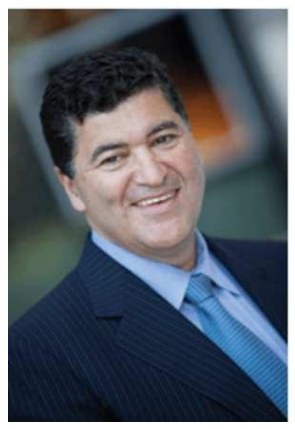

Translational and Clinical Science - Time

for a New

Vision

Figure 4. Dr. Elias Zerhouni, director of the National Institutes of Health (2002-2008) published a roadmap for NIH on Science, clarifying that translational medicine would be a core and mainstream of researches in the future [45].

In conclusion, this review discloses the beneficial educational impacts of 3D simulation and biomarkers which can increase the authenticity of learning, and also the learner's anatomical understanding of a disease process such as bone tumors. We can connect the orthopaedic dots better by understanding and adopting the TM models established elsewhere as discussed previously. In addition one could initiate their own TM community in most city and town regions, since colleges and universities are fairly numerous and open to collaborations with progressive clinicians (see illustrations presented earlier in this article). Partnerships can be established with local orthopaedic residency programs possibly as well as businesses and orthopedic companies. The local sales representatives can often connect surgeons with their respective R\&D personnel.

\section{Funding}

There is no funding

\section{Conflict of interest}

The author declares that there is no conflict of interest

\section{References}

[1] Geraghty J. Adenomatous polyposis coli and translational medicine. Lancet, 1996, 348, 422.

[2] Aoki KR, Ranoux D, Wissel J. Using translational medicine to understand clinical differences between botulinum toxin formulations. Eur J Neurol, 2006, 13: 10-19. 
[3] Kazdin AE. Evidence-based treatment and practice: new opportunities to bridge clinical research and practice, enhance the knowledge base, and improve patient care. Am Psychol, 2008, 63(3): 146-159.

[4] Mediouni M, Schlatterer DR, Madry $\mathrm{H}$ et al. A review of translational medicine. The future paradigm: how can we connect the orthopedic dots better? Curr Med Res Opin. 2018, 34(7):1217-1229.

[5] Schiffrin EL, Touyz RM. From bedside to bench to bedside: role of reninangiotensinaldosterone system in remodeling of resistance arteries in hypertension. Am J Physiol Heart Circ Physiol, 2004, 287: 435-446.

[6] Mankoff SP, Brander C, Ferrone S, Marincola FM. Lost in translation: obstacles to translational medicine. J Transl Med. 2004, 2: 14.

[7] Fontanarosa PB, DeAngelis CD. Basic science and translational research in JAMA. JAMA, 2002, 287: 1728.

[8] loannidis JP. Materializing research promises: opportunities, priorities and conflicts in translational medicine. J Transl Med. 2004, 2: 5.

[9] Sung NS, Crowley WF Jr, Genel M, et al. Central challenges facing the national clinical research enterprise. JAMA, 2003, 289: 1278-1287.

[10] Fontanarosa PB, DeAngelis CD. Translational medical research. JAMA, 2003, 289: 2133.

[11] Schechter AN, Perlman RL, Rettig RA. Why is revitalizing clinical research so important, yet so difficult? Perspect Biol Med. 2004, 47: 476-486.

[12] Mediouni M, Schlatterer DR. Frailty as an Outcome Predictor After Ankle Fractures: Where Are We Now? Geriatr. Orthop. Surg. Rehabil. 2018,9: 1-3.

[13] Knutsen G, Drogset JO, Engebretsen L, et al. A Randomized Multicenter Trial Comparing Autologous Chondrocyte Implantation with Microfracture: Long-Term Follow-up at 14 to 15 Years. J Bone Joint Surg Am, 2016, 98: 1332-1339.

[13] Gawande AA. Creating the educated surgeon in the 21st century. Am J Surg. 2001, 181:551-556?

[14] Howell WS. The empathic communicator. Bellmont, CA: Wadsworth; 1982.

[15] Miller GE. The assessment of clinical skills/competence/performance. Acad Med 1900;65:S63e7.

[16] Hazim Sadideen, Abtin Alvand, Munir Saadeddin, Roger Kneebone. Surgical experts: Born or made? International Journal of Surgery. 11 (2013) 773-778. [17] Mediouni M, A new generation of orthopaedic surgeons "T-model". Current Orthopaedic Practice. 2019 30:444-445.

[18] Mediouni M, Volosnikov A. The trends and challenges in orthopaedic simulation. J Orthop, 2015, 12: 253-259.

[19] Mediouni M, Kucklick T, Poncet S, Madiouni R, Abouaomar A, Madry M, Cucchiarini M, Chopko B, Vaughan N, Arora M, Gökkus K, Lozoya Lara M, Paiva Cedeño L, Volosnikov A, Hesmati M \& Ho K. An overview of thermal necrosis: present and future. Curr Med Res Opin. 2019, 9: 1555-1562.

[20] Mediouni M, Schlatterer DR, Khoury A, et al. Optimal Parameters to Avoid Thermal Necrosis During Bone Drilling: A Finite Element Analysis. J Orthop Res, 2017, 35: 2386-2391. 
[21] Zhao K, Malhotra P, Rosen D, Dalton P, Pribitkin EA. Computational Fluid Dynamics (CFD) as surgical planning tool: a pilot study on middle turbinate resection. Anat Rec (Hoboken), 2014, 297:2187-2195.

[22] Goldblatt EM, Lee WH. From bench to bedside: the growing use of translational research in cancer medicine. Am J Transl Res, 2010, 2:1-18.

[23] Dyke JP, Panicek DM, Healey JH, et al. Osteogenic and Ewing sarcomas: estimation of necrotic fraction during induction chemotherapy with dynamic contrast-enhanced MR imaging. Radiology, 2003, 228: 271-278.

[24] Paldino MJ, Barboriak DP. Fundamentals of Quantitative Dynamic ContrastEnhanced MR Imaging. Magn Reson Imaging Clin N Am. 2009, 17:277-289.

[25] Hayashida Y, Yakushiji T, Awai K, et al. Monitoring therapeutic responses of primary bone tumors by diffusion-weighted image: initial results. Eur Radiol, 2006, 16: 2637-2643.

[26] Van der Meel R, Gallagher WM, Oliveira S, O'Connor AE, Schiffelers RM, Byrne AT. Recent advances in molecular imaging biomarkers in cancer: application of bench to bedside technologies. Drug Discov Today, 2010,15: 102-114.

[27] Hwang S, Panicek DM. The evolution of musculoskeletal tumor imaging. The evolution of musculoskeletal tumor imaging. Radiol Clin North Am, 2009, 47:435-453. [28] Espina V, Dettloff KA, Cowherd S, Petricoin 3rd EF, Liotta LA. Use of proteomic analysis to monitor responses to biological therapies. Expert Opin Biol Ther, 2004, 4:83-93.

[29] Gehrke T, Parvizi J. Proceedings of the International Consensus Meeting on Periprosthetic Joint Infection. J Arthroplasty 2014;29:1-4.

[30] Xiong L, Li S, Dai M. Comparison of D-dimer with CRP and ESR for diagnosis of periprosthetic joint infection. J Orthop Surg Res. 2019 Jul 29;14(1):240.

[31] Ettinger $M$, Savov $P$, Calliess $T$, Windhagen $H$, Lichtinghagen $R$, Lukasz A, Omar $M$. Improved diagnostic accuracy with the classification tree method for diagnosing low-grade periprosthetic joint infections by quantitative measurement of synovial fluid alpha-defensin and C-reactive protein. Int Orthop. 2019 May 24.

[32] Shahi A, Parvizi J. The role of biomarkers in the diagnosis of periprosthetic joint infection. EFORT Open Rev. 2017 Mar 13;1(7):275-278.

[33] Ross RD, Deng Y, Fang R, Frisch NB, Jacobs JJ, Sumner DR. Discovery of biomarkers to identify peri-implant osteolysis before radiographic diagnosis. J Orthop Res. 2018 Oct;36(10):2754-2761.

[34] Anderson DD, Chubinskaya S, Guilak F, et al. 2011. Posttraumatic osteoarthritis: improved understanding and opportunities for early intervention. J Orthop Res

29:802-809.

[35] Goldring MB, Goldring SR. 2007. Osteoarthritis. J Cell Physiol 213:626-634.

[36] Loeser RF. 2010. Age-related changes in the musculoskeletal system and the development of osteoarthritis. Clin Geratr Med 26:371-386.

[37] Mcllwraith CW, Kawcak CE, Frisbie DD, Little CB, Clegg PD, Peffers MJ, Karsdal MA, Ekman S, Laverty S, Slayden RA, Sandell $L$, Lohmander LS, Kraus VB. Biomarkers for equine joint injury and osteoarthritis. J Orthop Res. 2018 Mar;36(3):823-831.

[38] K. Ochi, Y. Daigo, T. Katagiri et al., "Prediction of response to neoadjuvant chemotherapy for osteosarcoma by geneexpression profiles," International Journal of Oncology, vol. 24, no. 3, pp. 647-655, 2004. 
[39] M. B. Mintz, R. Sowers, K. M. Brown et al., "An expression signature classifies chemotherapyresistant pediatric osteosarcoma," Cancer Research, vol. 65, no. 5, pp. 1748-1754, 2005.

[40] P. Leonard, T. Sharp, S. Henderson et al., "Gene expression array profile of human osteosarcoma," British Journal of Cancer, vol. 89, no. 12, pp. 2284-2288, 2003.

[41] Mediouni M Kaczor_Urbanowicz EK, Madiouni R. Suggestions for creating the International Scientific Foundation of Saliva Diagnosis (ISFSD): New research strategies, development, and technologies. Oral Science International 2019.

[42] Panchmatia, J. R., Visenio, M. R., \& Panch, T. (2018). The role of artificial intelligence in orthopaedic surgery. British Journal of Hospital Medicine, 79(12), 676681.

[43] Langerhuizen, D. W., Janssen, S. J., Mallee, W. H., van den Bekerom, M. P., Ring, D., Kerkhoffs, G. M., ... \& Doornberg, J. N. (2019). What are the applications and limitations of artificial intelligence for fracture detection and classification in orthopaedic trauma imaging? A Systematic Review. Clinical Orthopaedics and Related Research®.

[44] Mediouni M, Schlatterer DR. Orthopaedic tumors: What problems are we solving, and are universities and major medical centers doing enough?. J Orthop. 2017;14(2):319-321.

[45] Zerhouni E. The NIH roadmap. Science. 2003;302:63-72

[46] Mediouni M, Schlatterer Dr \& Khoury A. Revisiting an old strategy: cartoons in medical education. Journal of Visual Communication in Medicine. 42(1), 2019. 